\title{
CLASSIFICATION OF METHODS FOR RISK MEASURES VAR AND CVAR CALCULATION AND ESTIMATION
}

\author{
N.G. ZRAZHEVSKA, A.G. ZRAZHEVSKY
}

\begin{abstract}
A systematic classification of the existing approaches for popular risk measures VaR and CVaR calculating and estimating is fulfilled. A review of the most used methods is done. For convenience, the considered methods are reduced to common econometric designations and concepts, guidance on the use of the methods is proposed. The correctness of the considered methods is numerically confirmed.

Keywords: estimation, value-at-risk, conditional value-at-risk, structural-hierarchical scheme, systematization, classification.
\end{abstract}

\section{INTRODUCTION}

Financial and economic crises of the end of the XX-beginning of the XXI century shows the necessity of further development of the risk theory. Determination and estimation of the possible risk arising from operational, financial and other activity of the company are among the main objectives of the risk management. Risk measures $\mathrm{VaR}$ and $\mathrm{CVaR}$ are widely used to solve this problem.

There are many works dedicated to risk measure VaR. They analyze its properties, advantages and disadvantages, the methods for its estimating [1-4 ]. VaR has become a standard, widely used risk measure because of its conceptual simplicity, ease of calculation and the availability of a sufficiently large number of standardized formulas and methods for calculation. At the same time, this risk measure has two major drawbacks: VaR is not coherent in the sense of [5], it does not have the sub-additivity property and $\mathrm{VaR}$ does not allow to determine the size of the potential losses that exceed the given level [6]. CVaR (ConditionalVaR) or, as it is called ES (Expected Shortfall), has been introduced to solve these problems $[5,6]$. Unlike VaR, CVaR is a coherent measure. Being a convex function, CVaR can be used in the optimization procedures [6]. The article [7] describes the methods, based on analytical expressions for $\mathrm{CVaR}$ calculation. The articles [8,9] provide a detailed comparative analysis of risk measures $\mathrm{VaR}$ and CVaR. The large number of approaches and methods complicates the selection of the optimal way for solving the problem. Systematization, classification and comparison of different methods for risk measures evaluation lead to the use of systematic methodology [10].

This paper discusses the main approaches for risk measures $\mathrm{VaR}$ and $\mathrm{CVaR}$ evaluation for a random variable, based on different statistical and econometric methods. Prices and other characteristics of financial instruments are usually considered as random variables. It allows to use random models.

The methods of risk measures estimations may be classified on the basis of statistical and stochastic approaches. This paper proposes the structural - hierarchical scheme (Fig. 1) with classification of the most popular methods. The scheme helps the user to choose a particular method. 


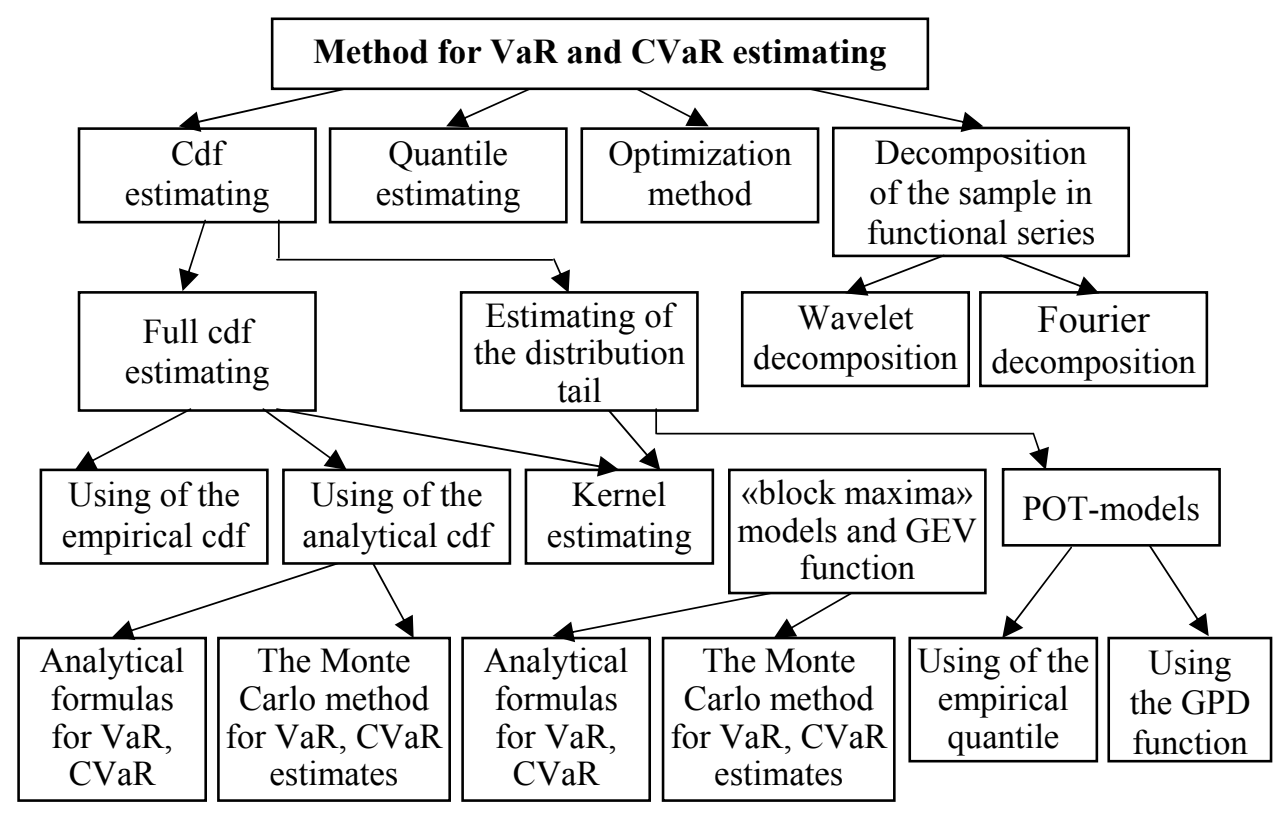

Fig.1. The structural - hierarchical scheme for VaR and CVaR estimating

Analysis of methods for VaR and CVaR estimating allowed to formulate the decision-making procedure for the choice of the method of static VaR and CVaR evaluation, depending on the research objectives and the characteristics of the analyzed data (Fig. 2).

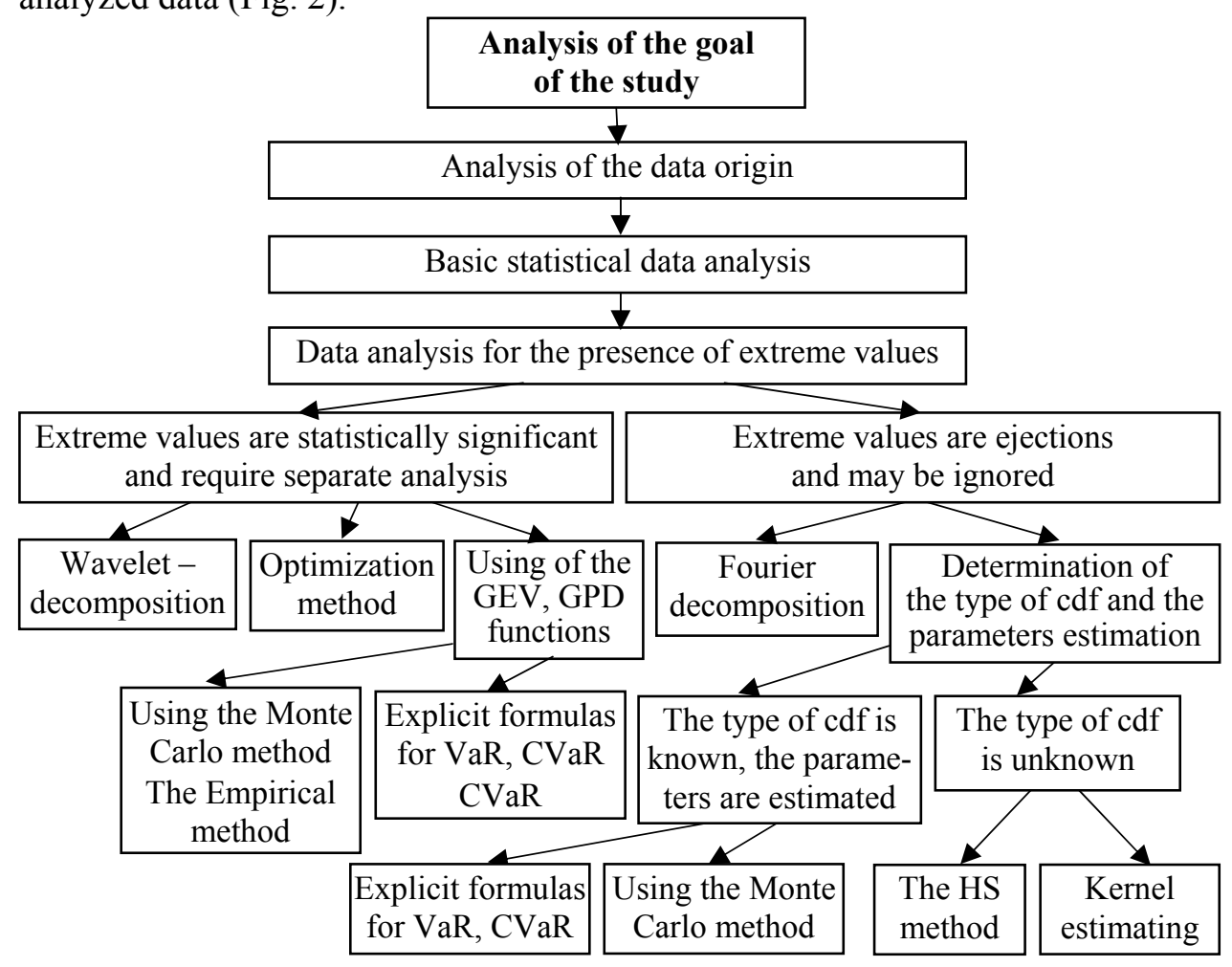

Fig. 2. The decision-making procedure for the choice of the method of static VaR and CVaR evaluation 
Sections 2-4 contain the mathematical methods that we use to concretize the methods of static VaR and CVaR estimating (Fig.1). Sections 5 and 6 show the results of numerical testing of the methods and the comparative analysis of the results.

\section{KEY DEFINITIONS}

Let $Y$ be a random variable describing the portfolio returns. For a given confidence level $\alpha \in(0 ; 1)$ let us consider the $(1-\alpha)$ th quantile of the returns distribution:

$$
y^{(1-\alpha)}(Y)=\sup \{y \mid P(Y \leq y) \leq 1-\alpha\} .
$$

From the viewpoint of econometric $y^{(1-\alpha)}(Y)$ determines the minimum value of the returns $Y$ with probability $(1-\alpha)$. If, for example, $\alpha=0,95$ then $y^{(1-\alpha)}(Y)$ (may be negative) with probability $95 \%$, determines the minimum returns. For ease of understanding econometrics operate with the concept $\operatorname{VaR}_{\alpha}(Y)$, defined as:

$$
\operatorname{VaR}_{\alpha}(Y)=-y^{(1-\alpha)}(Y) \text {. }
$$

So, $\operatorname{VaR}_{\alpha}(Y)$ with probability $(1-\alpha)$ defines the limit value of the loss (the sign «-»).

Some works operate with losses. Let $X$ denotes a random variable describing the portfolio losses. Then $X=-Y$, the quantile of the cumulative distribution function (cdf) of the loss function is defines as $x^{(\alpha)}(X)=\inf \{x \mid P(X \leq x) \geq \alpha\}$ and

$$
\operatorname{VaR}_{\alpha}(X)=x^{(\alpha)}(X) .
$$

So $\operatorname{VaR}_{\alpha}(Y)=\operatorname{VaR}_{\alpha}(X), x^{\alpha}(X)=-y^{(1-\alpha)}(Y)$.

From the viewpoint of statistics from (1) we see that:

$$
\operatorname{VaR}_{\alpha}(X)=F_{X}^{-1}(\alpha),
$$

where $F_{X}^{-1}(\cdot)$ is the inverse function of the cdf of the random variable $X$.

For a given confidence level $\alpha \in(0 ; 1)$ the risk measure $\mathrm{CVaR}_{\alpha}(X)$ can be defined as an average expected value of the loss with probability $\alpha$ :

$$
\mathrm{CVaR}_{\alpha}(X)=E\left[X \mid X>\operatorname{VaR}_{\alpha}(X)\right] .
$$

In the case if $\operatorname{VaR}_{\gamma}(X),(\gamma \in(0 ; \alpha))$ is integrability, $\operatorname{CVaR}_{\alpha}(X)$ may be defined as:

$$
\operatorname{CVaR}_{\alpha}(X)=\frac{1}{1-\alpha} \int_{\alpha}^{1} \operatorname{VaR}_{\gamma}(X) d \gamma .
$$

The paper [11] gives the following definition of CVaR: 


$$
\begin{aligned}
& \operatorname{CVaR}_{\alpha}(X)=\frac{1}{\alpha}\left[E\left[X \cdot \mathrm{I}\left\{X \leq \operatorname{VaR}_{\alpha}(X)\right\}\right]+\right. \\
& \left.+\alpha \operatorname{VaR}_{\alpha}(X)-\operatorname{VaR}_{\alpha}(X) P\left(X \leq \operatorname{VaR}_{\alpha}(X)\right)\right],
\end{aligned}
$$

where $I\{\}$ denotes the indicator function.

Consider an alternative definition of the risk measures [6]. Let $X$ be a random variable with the probability density function (pdf) $p(x), \xi \in R$ is a scalar, the function $f(\xi, x)$ for each fixed $\xi$ is a random variable with pdf $p(x)$. Let us introduce the function $\psi(\xi, \gamma)$ - the probability that $f(\xi, x)$ will not exceed the given level $\gamma$ :

$$
\psi(\xi, \gamma)=\int_{f(\xi, x) \leq \gamma} p(x) d x .
$$

Here $\psi(\xi, \gamma)$ is a loss distribution function. Then $\operatorname{VaR}_{\alpha}(X)$ and $\mathrm{CVaR}_{\alpha}(X)$ may be defined as:

$$
\begin{gathered}
\operatorname{VaR}_{\alpha}(X)=\min \{\gamma \in R \mid \psi(\xi, \gamma) \geq \alpha\}, \\
\operatorname{CVaR}_{\alpha}(X)=\frac{1}{1-\alpha} \int_{f(\xi, x) \geq \operatorname{VaR}_{\alpha}(X)} f(\xi, x) p(x) d x .
\end{gathered}
$$

Comments. A great number of mathematical and econometric studies on $\mathrm{VaR}$ and $\mathrm{CVaR}$, leads to a problem of a confidence level $\alpha$ choice. Note that the events «loss is larger than given level» and «loss do not achieve the level» are a complete group of events with probabilities $\alpha$ and $1-\alpha$ respectively. In this paper, the confidence level is the $\alpha$ th quantile of the loss function (1), measures of risk are designated as $\mathrm{VaR}_{\alpha}, \mathrm{CVaR}_{\alpha}$. In practice, the level is $\alpha \in[0,9 ; 1)$.

Nowadays there are many methods of VaR and CVaR estimating. In this paper we have no goal to review all of them. We propose a classification system of the best known and most commonly used approaches (Fig. 1) and briefly give the mathematical methods (description) that is required for the hierarchical scheme (Fig. 2) specification.

\section{THE METHODS OF VAR AND CVAR ESTIMATING USING THE CDF OF A RANDOM VARIABLE}

Let $X$ be a random variable. $X_{1}, X_{2}, \ldots, X_{n}$ is a sample of its values, $X_{(1)} \leq X_{(2)} \leq, \ldots, \leq X_{(n)}$ is the order statistics in ascending order. We assume that the distribution function of $X$ can be defined in an analytical or empirical form. Then definitions of VaR and CVaR (2) or (3) are used respectively.

\section{VaR and CVaR estimating using the full CDF}

\section{Methods with the use of the empirical CDF}

This group of methods includes, first of all, the Historical Simulation methods - HS [7]. This method is based on the construction of empirical distribution function using historical data. Then: 


$$
\begin{gathered}
\overline{\operatorname{VaR}}_{\alpha}=X_{([n \alpha] / n),} \\
\overline{\mathrm{CVaR}}_{\alpha}=\left(\sum_{i=[n \alpha]}^{n} X_{(i)}\right) /(n-[n \alpha]) .
\end{gathered}
$$

Estimate (7) can be modified. The paper [12] proposes the following estimate:

$$
\overline{\mathrm{CVaR}}_{\alpha}=\frac{1}{1+[n \alpha]} \sum_{i=1}^{n} X_{i} \mathrm{I}\left\{X_{i} \geq X_{([n \alpha]+1)}\right\}
$$

the paper [13] (for returns) gives the estimate:

$$
\overline{\mathrm{CVaR}}_{\alpha}=-\left(\frac{1}{[n(1-\alpha)]} \sum_{i=1}^{[n(1-\alpha)]} Y_{(i)}+\left(1-\frac{[n(1-\alpha)]}{n(1-\alpha)}\right) Y_{([n(1-\alpha)]+1)}\right) ;
$$

and the paper [14] (for returns):

$$
\overline{\mathrm{CVaR}}_{\alpha}=\left\{\begin{array}{c}
-\bar{Y}_{n(1-\alpha): \mathrm{n}, n}, n(1-\alpha) \in Z, \\
-\alpha \bar{Y}_{n(1-\alpha): \mathrm{n}}-(1-\alpha) \bar{Y}_{n(1-\alpha)+1: \mathrm{n}}, n(1-\alpha) \notin Z,
\end{array}\right.
$$

where $\bar{Y}_{k: n}=\frac{1}{k}\left(Y_{(1)}+\ldots+Y_{(k)}\right), k=\overline{1, n}$.

The advantage of the HS method is its simplicity in realization. Furthermore, the method does not require a prior assumptions about the type of the cdf. The HS method is very popular among economists and is implemented in many standard packages for risk measures calculating, such as Matlab, PSG. However, using the empirical cdf we suppose that the statistical characteristics of a random variable will be stable. Empirical distribution function is smooth enough in the vicinity of the mean value, and demonstrates jumps at the tails due to the relatively small number of extreme values in the sample. To overcome this problem the large volume samples must be analyzed. The Bank of International Settlements recommends to use samples consisting of not less than 250 data [15].

Let us consider the Rockafellar-Uryasev discrete method [16], based on the empirical distribution. Let for a random variable we have a variation row $X_{(1)} \leq X_{(2)} \leq \ldots \leq X_{(n)}$ with corresponding empirical probabilities $p_{k}>0$. Let $k_{\alpha}$ is a single index such that $\sum_{k=1}^{k_{\alpha}} p_{k} \geq \alpha>\sum_{k=1}^{k_{\alpha}-1} p_{k}$. Then:

$$
\operatorname{VaR}_{\alpha}=X_{\left(k_{\alpha}\right)}, \mathrm{CVaR}_{\alpha}=\frac{1}{1-\alpha}\left[\left(\sum_{k=1}^{k_{\alpha}} p_{k}-\alpha\right) X_{\left(k_{\alpha}\right)}+\sum_{k=k_{\alpha}+1}^{k_{\alpha}} p_{k} X_{(k)}\right] .
$$

\section{Methods with the use of the analitycal cdf}

If we can assume that a random variable has a certain type of cdf and we can evaluate the parameters of the distribution, analytic formulas can be used to find VaR and CVaR estimates. 
Let $X$ denote a standard normal random variable with mean $\mu$ and variance $\sigma^{2}$. Then [17]:

$$
\operatorname{VaR}_{\alpha}=\mu+\sigma \Phi^{-1}(\alpha), \operatorname{CVaR}_{\alpha}=\mu+\sigma \frac{\phi\left(\Phi^{-1}(\alpha)\right)}{1-\alpha} .
$$

Hereinafter, $\phi(\cdot)$ is the standard normal pdf and $\Phi(\cdot)$ is the standard normal cdf.

The standard normal pdf is symmetrical. At the same time, many financial instruments demonstrate the skewness of the distribution. The most popular and the most widely used of skewed extensions of the normal distribution is the skewnormal distribution due to Azzalini [18]. The cdf of this distribution is given by:

$$
F(x)=\Phi\left(\frac{x-\mu}{\sigma}\right)-2 T\left(\frac{x-\mu}{\sigma}, \lambda\right)
$$

where $x \in R, \mu \in R, \sigma>0, \lambda \in R, T(h, a)=\frac{1}{2 \pi} \int_{0}^{a} \frac{\exp \left\{-h^{2}\left(1+x^{2}\right) / 2\right\}}{1+x^{2}} d x$.

For a skew normal random variable, $\mathrm{VaR}_{\alpha}$ is defined as the unique root of the equation $F(x)=\alpha$, and the $\mathrm{CVaR}_{\alpha}$ can be expressed as:

$$
\mathrm{CVaR}_{\alpha}=\mu+\frac{\delta \sqrt{2}}{\alpha \sqrt{\pi}}\left[\lambda \Phi\left(z_{\alpha}\right)-\sqrt{2 \pi} \phi\left(y_{\alpha}\right) \Phi\left(\lambda y_{\alpha}\right)\right],
$$

where $\delta=\lambda / \sqrt{1+\lambda^{2}}, y_{\alpha}=\frac{V a R_{\alpha}-\mu}{\sigma}, z_{\alpha}=\sqrt{1+\lambda^{2}} y_{\alpha}$.

If $X$ is a random variable with the pdf: $f(x)=\frac{2}{\sigma} \sum_{i=1}^{L} \eta_{i} \varphi\left(\frac{x-\mu_{i}}{\sigma_{i}}\right) \times$ $\times \Phi\left(\lambda_{i} \frac{x-\mu_{i}}{\sigma_{i}}\right)$, where the weights $\eta_{i}$ are non-negative and sum to one $\left(\eta_{i} \geq 0, \sum_{i=1}^{L} \eta_{i}=1\right)$, for $\mathrm{CVaR}_{\alpha}$ takes place the formula:

$$
\mathrm{CVaR}_{\alpha}=\sum_{i=1}^{L} \pi_{i}\left\{\mu_{i}+\frac{\sigma_{i} \sqrt{2}}{\alpha \sqrt{\pi}}\left[\lambda_{i} \Phi\left(z_{\alpha, i}\right)-\sqrt{2 \pi} \phi\left(y_{\alpha, i}\right) \Phi\left(\lambda_{i} y_{\alpha, i}\right)\right]\right\},
$$

where $\sigma_{i}=\frac{\lambda_{i}}{\sqrt{1+\lambda_{i}^{2}}}, z_{\alpha, i}=\sqrt{1+\lambda_{i}^{2}} y_{\alpha, i}, y_{\alpha, i}=\frac{x_{\alpha, i}-\mu_{i}}{\sigma_{i}}, x_{\alpha, i}$ is the root of

$$
\begin{gathered}
\Phi\left(\frac{x-\mu_{i}}{\sigma_{i}}\right)-2 T\left(\frac{x-\mu_{i}}{\sigma_{i}}, \lambda_{i}\right)=\alpha, \\
\pi_{i}=\frac{\eta_{i}}{\alpha}\left[\Phi\left(\frac{\mathrm{VaR}_{\alpha}-\mu_{i}}{\sigma_{i}}\right)-2 T\left(\frac{\mathrm{VaR}_{\alpha}-\mu_{i}}{\sigma_{i}}, \lambda_{i}\right)\right] .
\end{gathered}
$$

As before $\mathrm{VaR}_{\alpha}$ is the root of $F(x)=\alpha$. 
Consider another version of representation of analytic formulas for VaR and CVaR.

Consider a random variable $\bar{X}=\frac{X-\mu}{\sigma}$, where a random variable $X$ has $t$-local-scailed distribution with parameters $\mu, \sigma$ and $v>2$ degrees of freedom, $\bar{X}$ has the standard Student's $t$ distribution with $v$ degrees of freedom and

$$
\begin{aligned}
E(\bar{X})= & 0, \operatorname{var}(\bar{X})=\frac{v}{v-2} . \text { Then [17]: } \\
& \operatorname{VaR}_{\alpha}=\mu+\sigma t_{v}^{-1}(\alpha), \operatorname{CVaR}_{\alpha}=\mu+\sigma \frac{g_{v}\left(t_{v}^{-1}(\alpha)\right)}{1-\alpha} \cdot \frac{v+\left(t_{v}^{-1}(\alpha)\right)^{2}}{v-1},
\end{aligned}
$$

where $g_{v}(\cdot), t_{v}(\cdot)$ denote, respectively, the pdf and the cdf of a Student's t random variable.

To take into account skewed of the pdf the article [19] proposes the Azzalini's skewed $t$ distribution. This distribution is given by the pdf

$$
f(x)=2 t_{\mathrm{v}}(x) g_{\mathrm{v}+1}\left(\lambda x \sqrt{\frac{\mathrm{v}+1}{x^{2}+v}}\right), x \in R ; v>0 .
$$

Let $F(\mathrm{x})$ be a corresponding cdf. Then $\mathrm{VaR}_{\alpha}$ is the root of $F(\mathrm{x})=\alpha$, and $\mathrm{CVaR}_{\alpha}=2 \int_{V a R_{\alpha}}^{+\infty} x t_{\mathrm{v}}(x) g_{\mathrm{v}+1}\left(\lambda x \sqrt{\frac{\mathrm{v}+1}{x^{2}+v}}\right) d x$.

A more complete list of analytical formulas for $\mathrm{VaR}$ and $\mathrm{CVaR}$, for different types of pdf can be found in [7].

The advantage of this approach is the availability of analytical formulas for risk measures calculating. However, the definition of the type of the pdf and estimation of its parameters may require considerable efforts.

\section{Monte Carlo and Richardson's method for risk measures estimating}

If we know the type of a random variable distribution and can estimate its parameters the Monte Carlo method can be used for VaR and CVaR estimating. The Monte Carlo method is based on the obtaining of a large number of realizations of a random variable such that their probability characteristics coincide with the estimates obtained by other methods. The principal difference between the Monte Carlo method and the method of historical simulation is that the original sample is generated on the basis of a model.

The Richardson's method for $\overline{\mathrm{CVaR}}_{\alpha}$ estimating on the basis of the Monte Carlo method is described in [7]. The method is formulated as the following algorithm.

1. Using the known cdf generate samples $X_{1}, \ldots, X_{N}$ by the Monte Carlo method.

2. Estimate the $\overline{\mathrm{CVaR}}_{\alpha}^{1}$ by the HS method. 
3. Repeat steps 1 and $2 \mathrm{M}$ times and compute the estimates $\overline{\mathrm{CVaR}}_{\alpha}^{j}$, $i=\overline{1, M}$ ( $M$ is the number of simulated samples).

4. Compute the mean: $m_{N}=\frac{1}{M} \sum_{i=1}^{M} \overline{\mathrm{CVaR}}_{\alpha}^{i}$.

5. Set $S_{n}=m_{N_{n}}, n=\overline{1, k+1}$ for some $k$ and $N_{1}, N_{2} \ldots N_{k+1}$.

6. Estimate $\overline{\mathrm{CVaR}}_{\alpha}$ as:

$$
\overline{\mathrm{CVaR}}_{\alpha}=\frac{\left|\begin{array}{cccc}
S_{1} & S_{2} & \ldots & S_{k+1} \\
1 & 1 / 2 & \ldots & 1 /(k+1) \\
. & . & . & . \\
1 & 1 / 2 & \ldots & 1 /(k+1)
\end{array}\right|}{\left|\begin{array}{cccc}
1 & 1 & \ldots & 1 \\
1 & 1 / 2 & \ldots & 1 /(k+1) \\
. & . & . & . \\
1 & 1 / 2 & \ldots & 1 /(k+1)
\end{array}\right|}
$$

\section{Kernel method}

The kernel method and its modifications are nonparametric methods of pdf estimation. They are based on the integral smoothing with a given kernel of empirical histogram and do not require a priori information about the type of distribution. The advantage of kernel methods is their independence from any information about the data source, as well as the availability of simple semi-analytic expressions for the estimates [12].

Let $K(\cdot)$ denote a symmetric kernel, smoothing function $G(t)=\int_{t}^{\infty} K(u) d u$, $G_{h}(t)=G(t / h)$, where $h$ is a suitable bandwidth. Then the smoothed estimate of the loss distribution function is $\bar{F}_{X}(x)=1-S_{h}(x)$, where $S_{h}(x)=$ $=\frac{1}{n} \sum_{t=1}^{n} G_{h}\left(x-X_{t}\right)$. The kernel estimation for $\overline{\mathrm{VaR}}_{\alpha}$ is a root of equation: $S_{h}(x)=1-\alpha$, and $\overline{\mathrm{CVaR}}_{\alpha}$ estimation can be written as:

$$
\overline{\mathrm{CVaR}}_{\alpha}=\frac{1}{n(1-\alpha)} \sum_{t=1}^{n} X_{t} G_{h}\left(\overline{\operatorname{VaR}}_{\alpha \alpha}-X_{t}\right) .
$$

The Trimmed kernel method [7] gives the following estimate for $\overline{\mathrm{CVaR}}_{\alpha}$. Let $X_{t}^{+}=X_{t} I\left\{\mathrm{X}_{t}>0\right\}$, a sequence $\left\{k_{n}\right\}$ is such that, $k_{n} \rightarrow \infty$ and $\frac{k_{n}}{n} \rightarrow 0$, $n \rightarrow \infty$. Then:

$$
\overline{\mathrm{CVaR}}_{\alpha}=\frac{1}{n(1-\alpha)} \sum_{i=1}^{n} X_{i} I\left\{\overline{\operatorname{VaR}}_{\alpha}(X) \leq X_{i} \leq X_{\left(\mathrm{k}_{n}\right)}^{+}\right\} .
$$




\section{Application of the extreme value theory to the risk measures var and cvar assessment}

As VaR is the quantile (and CVaR is the mean quantile), it is sufficient to study only the right tail of the pdf to get their estimations. Very often loss distributions are skewed and have so-called fat tails. In this case, the use of methods based on a priori assumptions about the normal distributions is untenable, and it makes sense to use Extreme Value Theory (EVT). EVT allows to analyze the extreme, and therefore relatively rare events in the historical data array.

Describe the mathematical formulation of EVT ([20]). Let we have a random variable $\mathrm{X}$ with cdf $\mathrm{F}(x)$, its sample $\left(X_{1}, \ldots, X_{n}\right)$ and $M_{n}=\max \left\{X_{1}, \ldots, X_{n}\right\}$. The EVT goal is to find a function $G(x)$, such that, $P\left(\frac{M_{n}-b_{n}}{a_{n}} \leq x\right) \rightarrow G(x)$, $n \rightarrow \infty$, where $\left\{a_{n}>0\right\},\left\{b_{n}\right\}$ are the sequences of constants. According to the Fisher-Tippett theorem, function $G(x)$ belongs to the generalized extreme value (GEV) family of distributions. These distributions have the form:

$$
G_{\mu, \sigma, \xi}(x)=\left\{\begin{array}{l}
\exp \left(-\left(1+\xi \frac{x-\mu}{\sigma}\right)^{-\frac{1}{\xi}}\right), \xi \neq 0 \\
\exp \left(-\exp \left(-\frac{x-\mu}{\sigma}\right)\right), \quad \xi=0
\end{array}\right.
$$

GEV involves three distributions: the Frechet distribution with «fat» tail if $\xi>0$, the Gambela distribution with «thin» tail if $\xi<0$ and the Weibull distribution if $\xi=0$. The parameter $\alpha=1 / \xi$ is called the tail index (for $\xi>0$ ).

A popular method of parameter $\xi$ estimating is the Hill's non-parametric method [20]. Consider a sample of losses and define the order statistics as $X_{(1)} \leq X_{(2)} \leq \ldots \leq X_{(n)}$. Then for integer $l>0$ we have the estimation for $\xi$ as: $\widehat{\xi}^{\text {Hill }}(l)=\frac{1}{l} \sum_{j=1}^{l}\left(\ln X_{(n-j+1)}-\ln X_{(n-l)}\right)$. It can be shown [3], that $\sqrt{k}\left|\xi^{H i l l}(l)-\xi\right|$ is asymptotically normally distributed $N\left(0 ; \xi^{2}\right)$. Parameter $l$ can be found from the plot $\left(k, \hat{\xi}^{\text {Hill }}(l)\right)$ : we select the value of $l$ such that the evaluation $\xi^{H i l l}(l)$ appears stable. A more accurate method for finding $l$ using the bootstrap procedure is described in [21]

The following formulas for risk measures estimating can be used [22]:

$$
\mathrm{VaR}_{\alpha}=\exp \left(-\left(\frac{l_{n, \alpha}}{n(1-\alpha)}\right)^{\hat{\xi}} X_{\left(l_{n, \alpha}\right)}\right)-1
$$




$$
\mathrm{CVaR}_{\alpha}=\frac{1}{1-\alpha} \int_{\alpha}^{1} \exp \left(-\left(\frac{l_{n, \alpha}}{n q}\right)^{\hat{\xi}} X_{\left(l_{n, \alpha}\right)}\right) d q-1,
$$

where $l_{n, \alpha}=[n(1,05-\alpha)]$.

\section{Block maxima method}

According to the block maxima method [20], the sample is divided into $m$ non-overlapping subsamples. For each subsample we find the maximum value $M_{n}^{(i)}, i=\overline{1, m}$. It is assumed that $m$ is sufficiently large so that the Fisher-Tippett theorem holds for $\left\{M_{n}^{(i)}\right\}$ and the function $G E V_{\mu, \xi, \sigma}(x)$ is the distribution function of maximum values in the sample. To find the estimates of parameters $\mu, \sigma, \xi$ maximum likelihood method can be used. Knowing the $G E V_{\mu, \xi, \sigma}(x)$ distribution the Monte Carlo method or analytical formulas can be used. For example the article [23] presents the next formulas:

$$
\begin{gathered}
\operatorname{VaR}_{p}=\mu-\frac{\sigma}{\xi}\left[1-\{-\ln \alpha\}^{-\xi}\right], \\
\mathrm{CVaR}_{p}=\frac{1}{\sigma} \int_{\operatorname{VaR}_{p}}^{+\infty} x\left(1+\xi \frac{x-\mu}{\sigma}\right)^{-1 / \xi-1} \exp \left\{-\left(1+\xi \frac{x-\mu}{\sigma}\right)^{-1 / \xi}\right\} d x,
\end{gathered}
$$

where $p=1+(\alpha-1) \frac{m}{n}$.

The disadvantage of the block maxima method is a potential shortage of extreme values because a single value is determined in each block. The POT (Peaks Over Thresholds) method uses more complete information about the extreme values.

\section{POT-method}

In the POT method [20] we choose some high enough threshold $u$ and consider only the sample values above it: $X_{i}>u$. It can be shown [20], that for large enough $u$ the excess distribution $F_{u}(y)=\mathrm{P}\{X-u \leq y \mid x>u\}, y>0$ is well approximated by the Generalized Pareto Distribution (GPD) given as:

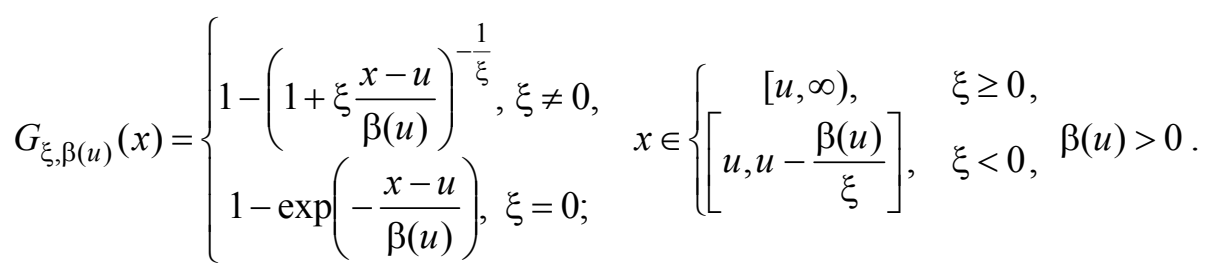

The advantage of the POT approach is that the method gives the explicit formulas for $\mathrm{VaR}$ and $\mathrm{CVaR}[20]$ :

$$
\overline{\operatorname{VaR}}_{\alpha}=u+\frac{\beta}{\xi}\left[\left(\frac{n(1-\alpha)}{k}\right)^{-\xi}-1\right], \overline{\mathrm{CVaR}}_{\alpha}=\frac{1}{1-\xi} \mathrm{VaR}_{\alpha}+\frac{\beta-\xi u}{1-\xi}
$$


The article [24] proposes the POT method with the use of the empirical distribution function. The main idea $f$ the method: the threshold value is displayed as a horizontal line on the plot of sampled values, connected successively. The extreme values are taken as the peak values between two distinct upcrossings. So we get the extreme value sample $\left\{X_{i}\right\}, i=\overline{1, N}$. To analyze the getting sample we divide the whole interval into $m$ intervals of the same length so that each interval has at least one peak. Then $n=\frac{N}{m}$ is the average number of peaks for one interval. So we can consider $n$ independent, identically distributed random variables, for which we have $N$ realizations. Then the cdf of all peaks above $u$ is:

$$
\bar{F}_{\xi}(x)=\prod_{i=1}^{n} P\left(X_{i} \leq x\right)=\left[F_{\text {pot }}(x)\right]^{n} .
$$

The function $F_{\text {pot }}(x)$ can be constructed as an empirical cdf and can be used for VaR and CVaR estimating.

\section{QUANTILE ESTIMATION}

Empirical quantile estimation is one of the classic nonparametric methods for $\mathrm{VaR}$ and $\mathrm{CVaR}$ estimating [3]. As before let $X$ be a random variable. $X_{1}, X_{2}, \ldots, X_{n}$ is a sample of its values, $X_{(1)} \leq X_{(2)} \leq \ldots \leq X_{(n)}$ is the order statistics in ascending order, $f(\mathrm{x})$ and $F(\mathrm{x})$ are the pdf and the cdf respectively. The method is based on the following theoretical result. If $x^{(\alpha)}$ is the $\alpha$ th quantile of $F(x)$ and $f\left(x^{(\alpha)}\right) \neq 0$ then

$$
X_{(l)} \sim N\left\{x^{(\alpha)}, \frac{\alpha(1-\alpha)}{n\left[f\left(x^{(\alpha)}\right)\right]^{2}}\right\}, l \rightarrow \infty,
$$

where $l=n \alpha$. Taking into account (1), the $\mathrm{VaR}_{\alpha}$ estimate can be obtained from (12) using $X_{(l)}$.

If $l=n \alpha$ is notinteger, the interpolation can be used for quantile estimation. Let $l_{1}, l_{2}$ be the two neighbouring positive integers such $l_{1}<n \alpha<l_{2}$ and $p_{i}=\frac{l_{i}}{n}, i=1,2$. Then:

$$
\begin{gathered}
\overline{\mathrm{VaR}}_{\alpha}=\frac{p_{2}-\alpha}{p_{2}-p_{1}} X_{\left(l_{1}\right)}+\frac{\alpha-p_{1}}{p_{2}-p_{1}} X_{\left(l_{2}\right)}, \\
\overline{\mathrm{CVaR}}_{\alpha}=\frac{1}{N_{\alpha}(1-\alpha)} \sum_{i=1}^{n} X_{(i)} I\left\{X_{(i)}>\overline{\operatorname{VaR}}_{\alpha}\right\},
\end{gathered}
$$

where $N_{\alpha}$ is the number of order statistics larger than $\overline{\mathrm{VaR}}_{\alpha}$. 
If the values of order statistics have different probabilities the risk measures estimates can be obtained as follows. We consider the order statistics:

$$
\begin{aligned}
& \left\{X_{(1)}, P_{(1)}\right\}, \ldots,\left\{X_{(n)}, P_{(n)}\right\}, \quad \sum_{i=1}^{n} P_{(i)}=1 . \text { Define } p=1+(\alpha-1) \frac{n}{N}, \bar{p}^{-}=\sum_{i=1}^{k^{-}} P_{(i)} \leq \\
& \leq p, \bar{p}^{+}=\sum_{i=1}^{k^{-}+1} P_{(i)} \geq p \text {. Then: } \\
& \overline{\operatorname{VaR}}_{\alpha}=X_{\left(k^{-}\right)}+\frac{X_{\left(k^{-}+1\right)}-X_{\left(k^{-}\right)}}{\bar{p}^{+}-\bar{p}^{-}}\left(p-\bar{p}^{-}\right), \\
& \overline{\mathrm{CVaR}}_{\alpha}=\overline{\operatorname{VaR}}_{\alpha} \cdot \frac{\bar{p}-\bar{p}^{-}}{\bar{p}^{+}-\bar{p}^{-}}+\frac{1}{n-k^{-}-1} \sum_{i=1}^{n-k-1} X_{\left(k^{-}+i\right)} P_{\left(k^{-}+i\right)} .
\end{aligned}
$$

Advantages of the empirical quantile estimation are its simplicity and using no specific distributional assumption. However it has some drawbacks. It assumes that the distribution under sample remains unchanged, that is often not so in practice. Furthermore, if $\alpha$ is close enough to 1 the empirical quantile is not efficient estimate of the theoretical quantile. In practice, $\overline{\mathrm{VaR}}_{\alpha}$ obtained by the empirical quantile can serve as a lower bound for tis risk measure.

\section{ROCKAFELLAR-URYASEV OPTIMIZATION METHOD}

Using the definition of $\mathrm{VaR}_{\alpha}$ and $\mathrm{CVaR}_{\alpha}$ in the form of (4) and (5), respectively, the paper [6] proposes the algorithm in the form of the optimization problem for risk measures estimating.

Define the function:

$$
F_{\alpha}(\xi, \beta)=\beta+\frac{1}{1-\alpha} \int_{x \in R}[f(\xi, x)-\beta]^{+} p(x) d x, \text { where }[t]^{+}=\left\{\begin{array}{l}
t, t>0 \\
0, t \leq 0 .
\end{array}\right.
$$

It is shown that $F_{\alpha}(\xi, \beta)$ is a convex and continuously differentiable at $\beta$ function. In this case:

$$
\operatorname{VaR}_{\alpha} \in \underset{\beta \in R}{\operatorname{argmin}} F_{\alpha}(\xi, \beta), \quad \mathrm{CVaR}_{\alpha}=\min _{\beta \in R} F_{\alpha}(\xi, \beta) .
$$

$\left(\operatorname{VaR}_{\alpha}(X)\right.$ is the left point of the solution set of $\left.\underset{\beta \in R}{\operatorname{argmin}} F_{\alpha}(\xi, \beta)\right)$.

The integral in (13) can be calculated approximately or exactly. For example, in the case of a discrete sample $\left\{X_{i}\right\}, i=\overline{1, n}$ the rectangles method will lead to the following formula: $F_{\alpha}(x, \beta)=\beta+\frac{1}{N(1-\alpha)} \sum_{k=1}^{N}\left[f\left(\xi, x_{k}\right)-\beta\right]^{+}$. To solve the optimization problems standard optimization packages such as PSG, Gurobi can be used. 
The optimization method can be used with practically no restrictions on the type of the distribution, the sample size, noisy empirical data. However, the use of optimization procedures requires high qualification of the user and access to specialized software (eg PSG). Unfortunately, the well-known packages Matlab and Mathematica allow to solve effectively only linear problems.

\section{APPLICATION OF METHODS OF VAR AND CVAR ESTIMATING`}

In this section we apply the methods described above for obtaining numerical values of VaR and CVaR for artificial data. The first group of data $(\mathrm{N})$ was modeled on assumption of normal distribution with parameters: $\mu=0,5 ; \sigma=5$. For the second group $(T)$ we use the $t$-local-scailed distribution with the parameters $\mu=0,5 ; \sigma=5, \nu=4.10,000$ values were generated in each group, the confidence level was taken as $\alpha=0,95 ; \alpha=0,99$. The results are shown in Tables 1,2 .

Table 1 shows the evaluations of the risk measures obtained with the use of the full cdf. The column Exact in the table shows the exact values (formulas (8), (9). As representatives of the methods based on the empirical distribution function we use the Historical Simulated method (formulas (6), (7)) - HS, and the Rockafellar-Uryasev discrete method ((9)) - R-U. Estimations PE we receive using (8), where the parameters of the normal distribution are estimated with the maximum likelihood method. Using the estimated distribution for the Monte Carlo method we get the estimates PEMC, and The Richardson's method gives us the estimates PEMCR.

Table 1. The results for $\mathrm{VaR}_{\alpha}$ and $\mathrm{CVaR}_{\alpha}$ estimating for different methods using the full cdf for different confidence levels

\begin{tabular}{|c|c|c|c|c|c|c|}
\hline Method/Risk & Exact & HS & R-U & PE & PEMC & PEMCR \\
\hline $\mathrm{VaR}_{0,95}(\mathrm{~N})$ & 8,72 & 8,57 & 8,57 & 8,52 & 8,35 & 8,50 \\
\hline $\mathrm{RE}$ & 0,00 & 0,02 & 0,02 & 0,02 & 0,04 & 0,03 \\
\hline $\mathrm{CVaR}_{0,95}(\mathrm{~N})$ & 10,81 & 10,57 & 10,57 & 10,58 & 10,49 & 10,56 \\
\hline $\mathrm{RE}$ & 0,00 & 0,02 & 0,02 & 0,02 & 0,03 & 0,02 \\
\hline $\mathrm{VaR}_{0,99}(\mathrm{~N})$ & 12,13 & 12,26 & 12,25 & 12,13 & 12,11 & 12,17 \\
\hline $\mathrm{RE}$ & 0,00 & $-0,01$ & $-0,01$ & 0,00 & 0,00 & 0,00 \\
\hline $\mathrm{CVaR}_{0,99}(\mathrm{~N})$ & 13,83 & 13,93 & 13,93 & 13,82 & 13,95 & 13,84 \\
\hline $\mathrm{RE}$ & 0,00 & $-0,01$ & $-0,01$ & 0,00 & $-0,01$ & 0,00 \\
\hline $\mathrm{VaR}_{0,95}(\mathrm{~T})$ & 11,16 & 10,99 & 10,98 & 11,02 & 10,95 & 11,05 \\
\hline $\mathrm{RE}$ & 0,00 & 0,02 & 0,02 & 0,01 & 0,02 & 0,01 \\
\hline $\mathrm{CVaR}_{0,95}(\mathrm{~T})$ & 16,51 & 15,95 & 15,95 & 16,03 & 16,00 & 16,11 \\
\hline $\mathrm{RE}$ & 0,00 & 0,03 & 0,03 & 0,03 & 0,03 & 0,02 \\
\hline $\mathrm{VaR}_{0,99}(\mathrm{~T})$ & 19,23 & 19,65 & 19,65 & 19,07 & 19,06 & 19,09 \\
\hline $\mathrm{RE}$ & 0,00 & $-0,02$ & $-0,02$ & 0,01 & 0,01 & 0,01 \\
\hline $\mathrm{C} \mathrm{VaR}_{0,99}(\mathrm{~T})$ & 26,60 & 26,67 & 26,67 & 26,17 & 26,92 & 26,12 \\
\hline $\mathrm{RE}$ & 0,00 & 0,00 & 0,00 & 0,02 & $-0,01$ & 0,02 \\
\hline
\end{tabular}


Table 2 shows the results obtained by the methods based on the analyzes of the tail of the distribution. Using the method of maximum likelihood we estimate the parameters for GEV (GPD) function. Estimates GEVQ (GPDQ) we get using formulas (10), (11), and the Monte Carlo method gives the estimates GEVMC (GPDMC). The empirical POT method gives POTE estimates.

$\mathrm{RE}$ means a relative error.

Ta b l e 2. The results for $\mathrm{VaR}_{\alpha}$ and $\mathrm{CVaR}_{\alpha}$ estimating for different methods using the tail of cdf for different confidence levels

\begin{tabular}{|c|c|c|c|c|c|}
\hline Method/Risk & GEVQ & GEVMC & GPDQ & GPDMC & POTE \\
\hline $\mathrm{VaR}_{0,95}(\mathrm{~N})$ & 7,89 & 7,88 & 8,83 & 8,90 & 8,71 \\
\hline $\mathrm{RE}$ & 0,10 & 0,10 & $-0,01$ & $-0,02$ & 0,00 \\
\hline $\mathrm{CVaR}_{0,95}(\mathrm{~N})$ & 10,33 & 10,28 & 10,96 & 10,92 & 10,71 \\
\hline $\mathrm{RE}$ & 0,05 & 0,05 & $-0,01$ & $-0,01$ & 0,01 \\
\hline $\mathrm{VaR}_{0,99}(\mathrm{~N})$ & 12,13 & 12,19 & 12,51 & 12,54 & 12,45 \\
\hline $\mathrm{RE}$ & 0,00 & $-0,01$ & $-0,03$ & $-0,03$ & $-0,03$ \\
\hline $\mathrm{CVaR}_{0,99}(\mathrm{~N})$ & 13,82 & 13,79 & 13,88 & 13,78 & 14,10 \\
\hline $\mathrm{RE}$ & 0,00 & 0,00 & 0,00 & 0,00 & $-0,02$ \\
\hline $\mathrm{VaR}_{0,95}(\mathrm{~T})$ & 10,96 & 10,91 & 11,72 & 11,83 & 11,08 \\
\hline $\mathrm{RE}$ & 0,02 & 0,02 & $-0,05$ & $-0,06$ & 0,01 \\
\hline $\mathrm{CVaR}_{0,95}(\mathrm{~T})$ & 15,92 & 15,95 & 16,60 & 16,49 & 16,06 \\
\hline $\mathrm{RE}$ & 0,04 & 0,03 & $-0,01$ & 0,00 & 0,03 \\
\hline $\mathrm{VaR}_{0,99}(\mathrm{~T})$ & 19,30 & 19,30 & 19,79 & 19,66 & 19,67 \\
\hline $\mathrm{RE}$ & 0,00 & 0,00 & $-0,03$ & $-0,02$ & $-0,02$ \\
\hline $\mathrm{CVaR}_{0,99}(\mathrm{~T})$ & 24,26 & 24,71 & 24,73 & 24,35 & 26,79 \\
\hline $\mathrm{RE}$ & 0,09 & 0,07 & 0,07 & 0,08 & $-0,01$ \\
\hline
\end{tabular}

All calculations performed with Matlab package. The results indicate the correctness of the formulas given in the article. The calculation error is caused by the limited sample and data discretization. It should be noted that the existence of analytical formulas allows to speed up the computation of risk measures, but does not significantly reduce the accuracy of the calculations. For real data results may vary, because of a priori hypotheses about the type of distributions (which can be wrong) and limited sample data.

\section{CONCLUSIONS}

The article systematizes and classifies the most common used methods of the risk measures $\mathrm{VaR}$ and $\mathrm{CVaR}$ calculation and estimation. In recent years, these risk measures have been used to analyze a wide class of data, that caused to a large number of different methodologies giving the ready formulas for their calculating or indicating an algorithm for estimating. However, in most studies, these methods are applied to concrete data with own specifics. That's why despite their universality VaR and CVaR have got a tough bind to specific data. In this paper we describe the methods without reference to the concrete data. 
Classification of methods presented in the form of a hierarchical table, that helps to determine the sequence of operations required to obtain VaR and CVaR values depending on the available information about analyzed data, the purpose of analysis and the availability of information and computing resources needed to get the result. Econometric concepts and designations are taken as a basis that helps to use the results of the article in solving applied problems. The analysis allowed to formulate the decision-making procedure for the choice of the method of static VaR and CVaR evaluation. The procedure includes all necessary steps to make a decision, from general statistical data analysis to the choice of a particular method for risk measures estimating.

\section{REFERENCES}

1. RiskMetrics. Technical Document, 4-th Edition / J.P. Morgan, December 1996. - $296 \mathrm{p}$.

2. Dowd K. Measuring Market Risk / K. Dowd // Chichester: John Wiley \& Sons, Inc., 2005. $-410 \mathrm{p}$.

3. Tsay R.S. Analysis of Financial Time Series / R.S. Tsay. — Hoboken: John Wiley \& Sons, Inc., 2010. $-714 \mathrm{p}$.

4. Embrechts $P$. Correlation and dependence in risk management: properties and pitfalls. Risk Management: Value at Risk and Beyond / P. Embrechts, A.J. McNeil, D. Straumann // Cambridge: Cambridge University Press, 2002. - P.176-223.

5. Artzner P. Coherent measures of risk / P. Artzner, F. Delbaen, J.M. Eber, D. Heath // Mathematical Finance. - 1999. — № 9. - P. 203-228.

6. Rockafellar R.T. Optimization of conditional value-at-risk / R.T. Rockafellar, S.P. Uryasev // Journal of Risk. — 2000. - 2. - P. 21-42.

7. Nadarajah $S$. Estimation methods for expected shortfall / S. Nadarajah, Zhang Bo, S. Chan // Quantitative Finance. - 2014. - 14. - P. 271-291.

8. Yamai Y. Comparative analysis of expected shortfall and value-at-risk: their estimation error, decomposition and optimization / Y. Yamai, T. Yoshiba // Monetary and Economic Studies. - 2002. - 20(1). - P. 57-86.

9. Sarykalin S. VaR vs.CVaR / S. Sarykalin, G. Serraino, S. Uryasev // Risk Management and Optimization Tutorialsin Operations Research INFORMS. 2008. - P. 270-294.

10. Згуровський М.З. Основи системного аналізу / М.З. Згуровський, Н.Д. Панкратова. - К.: ВНV, 2007. - $544 \mathrm{c}$.

11. Acerbi C. Expected Shortfall: a natural coherent alternative to Value at Risk / C. Acerbi, D. Tasche // Economic Notes. - 2002. - 31. - P. 379-388.

12. Chen S.X. Nonparametric estimation of expected shortfall / S.X. Chen // Journal of Financial Econometrics. - 2008. - 6. - P. 87-107.

13. Peracchi $F$. On estimating the conditional expected shortfall / F. Peracchi, A.V. Tanase // Applied Stochastic Models in Business and Industry. — 2008. - 24. P. 471-493.

14. Inui $K$. On the significance of expected shortfall as a coherent risk measure / K. Inui, M. Kijima // Journal of Banking and Finance. — 2005. - 29. P. $853-864$. 
15. Меньшиков И.С. Рыночные риски, модели и методы / И.С. Меньшиков, Д.А. Шелагин. - М.: Росс. академия наук. Вычислит. центр, 2006. - 55 с.

16. Rockafellar R.T. Conditional value-at-risk for general loss distributions / R.T. Rockafellar, S.P. Uryasev // Journal of Banking \& Finance. — 2002. — 26. — P. 1443-1471.

17. Kjellson B. Forecasting Expected Shortfall / B. Kjellson // Bachelor's Theses in Mathematical Sciences. - 2013. - K7. - P. 1-39.

18. Bernardi $M$. Risk measures for skew normal mixtures // Statistics \& Probability Letters. - 2013. - 83 (8). - P. 1819-1824.

19. Broda S.A. Expected shortfall for distributions in finance / S.A. Broda, M.S. Paolella // Statistical Tools for Finance and Insurance. — 2011. — P. 57-99.

20. Zivot E. Modeling Financial Time Series with S-PLUS / E. Zivot, J. Wang. - New York: Springer-Verlag, 2003. - $705 \mathrm{p}$.

21. Danielsson J. Value at Risk and Extreme Returns / J. Danielsson, C.G. de Vries // Annales d'economie et de Statistique. - 2000. - 60. - P. 236-269.

22. Embrechts $P$. Strategic long-term financial risks: single risk factors / P. Embrechts, R. Kaufmann, P. Patie // Computational Optimization and Applications. 2005. - 32. - P. 61-90.

23. $O u$ S. Robustness analysis and algorithm of expected shortfall based on extremevalue block minimum model / S. Ou, D. Yi // Proceedings of the 2009 International Conference. - 2009.

24. Rinker J. Peak-over-Threshold Method for Extreme Values / J. Rinker // Duke University. - 2013. - 22. - P. 1-6.

Resieved 14.06.2016

From the Editorial Board: the article corresponds completely to submitted manuscript. 